\title{
Mucocele de cavidades paranasales
}

\author{
Sinus mucocele \\ José Tomás Zemelman', Paula Ruz $\mathbf{M}^{1,2}$, Marcela Veloz $\mathrm{T}^{1,2}$, Samanta Ruz $\mathbf{G}^{1,3}$, Constanza Valdés $\mathrm{P}^{1,4}$.
}

\section{RESUMEN}

Los mucoceles son quistes expansivos e indolentes de las cavidades paranasales. A pesar de ser lesiones benignas, tienen potencial destructivo local por su expansión crónica y cambios óseos. Su ubicación más frecuente es frontoetmoidal. Se postula que su origen es por alteración de la vía de drenaje de los senos. La clínica es dependiente de su ubicación: los mucoceles frontoetmoidales presentan aumento de volumen, cefalea o proptosis. Las imágenes juegan un rol importante en el diagnóstico, siendo la tomografía computarizada y la resonancia magnética los exámenes que detectan patrones sugerentes de mucoceles. El tratamiento es quirúrgico, donde el abordaje endoscópico ha desplazado al abierto por ser mínimamente invasivo, presentar menos comorbilidades y tener menor tasa de recurrencia.

Palabras clave: Mucocele, cavidades paranasales, senos paranasales, manejo quirúrgico, endoscopía nasal, cirugía endoscópica nasal.

\begin{abstract}
Mucoceles are expansive and indolent cyst of the paranasal cavities. Despite being benign lesions, they have local destructive potential because of its chronic expansion and bony changes. Its most common location is frontoethmoidal. Alterations in the drainage pathway of sinus is thought to be the origin of mucoceles. The clinical features depend on the location. Frontoethmoidal often presents frontal swelling, headache or proptosis. Imaging plays an important part of diagnosis. Tomography and magnetic resonance have patterns that can suggest the presence of a mucocele. Paranasal sinus mucoceles are primarily treated surgically. The endoscopic surgical management has replaced the open resection because of its minimally invasive treatment, less morbidity and low recurrence rates.
\end{abstract}

Key words: Mucocele, paranasal sinuses, sinus cavity, nasal endoscopy, sinus surgery.

\footnotetext{
1 Servicio de Otorrinolaringología Hospital del Salvador, Universidad de Chile, Santiago, Chile.

2 Servicio de Otorrinolaringología Clínica Santa María, Santiago, Chile.

3 Servicio de Otorrinolaringología Clínica Indisa, Santiago, Chile.

4 Servicio de Otorrinolaringología Clínica Las Condes, Santiago, Chile.
}

Los autores declaran no tener conflictos de interés.

Recibido el 8 de septiembre de 2019. Aceptado el 7 de enero de 2020. 


\section{INTRODUCCIÓN}

Los mucoceles de cavidades paranasales (CPN) son quistes expansivos e indolentes compuestos por una pared externa de epitelio respiratorio normal y contenido mucoso aséptico. A pesar de ser lesiones benignas, tienen potencial destructivo local por su expansión crónica, remodelamiento y erosión ósea que provoca la producción continua de secreciones en su interior ${ }^{1}$. Su contenido mucoso tiene potencial de infectarse, denominándose piomucocele ${ }^{2}$.

\section{EPIDEMIOLOGÍA}

La edad de presentación de los mucoceles es de los 40 a los 60 años sin predilección por género ${ }^{3,4}$. Afectan de forma predominante al seno frontal, encontrándose ahí el $60 \%$ a $65 \%$ del total de mucoceles. Siguen en orden de frecuencia seno etmoidal con el $20 \%$ a $30 \%$, maxilares con $10 \%$ y raramente en esfenoides con $2 \%$ a $3 \%$ de los casos (Tabla 1). La presencia de mucoceles se ha descrito en áreas inusuales hasta en $2,3 \%$ de los casos, como en crista Galli, celdillas de Onodi, conchas bulosas de cornetes medios, celdillas de Haller y también en procesos uncinados ${ }^{5,6}$.

\section{FISIOPATOLOGÍA}

El origen de los mucoceles es controversial. Una de las teorías es que se originan a partir de alguna alteración en la anatomía de la vía de drenaje de los senos paranasales. La distribución, con predominio frontoetmoidal (Figura 1), estaría dada porque éstos son los senos con áreas

Tabla 1. Ubicación más frecuente de mucoceles en cavidades paranasales

\begin{tabular}{|lc|}
\hline Ubicación & Frecuencia \\
\hline Frontal & $60 \%-65 \%$ \\
Etmoidal & $20 \%-30 \%$ \\
Maxilar & $10 \%$ \\
Esfenoides & $2 \%-3 \%$ \\
\hline
\end{tabular}

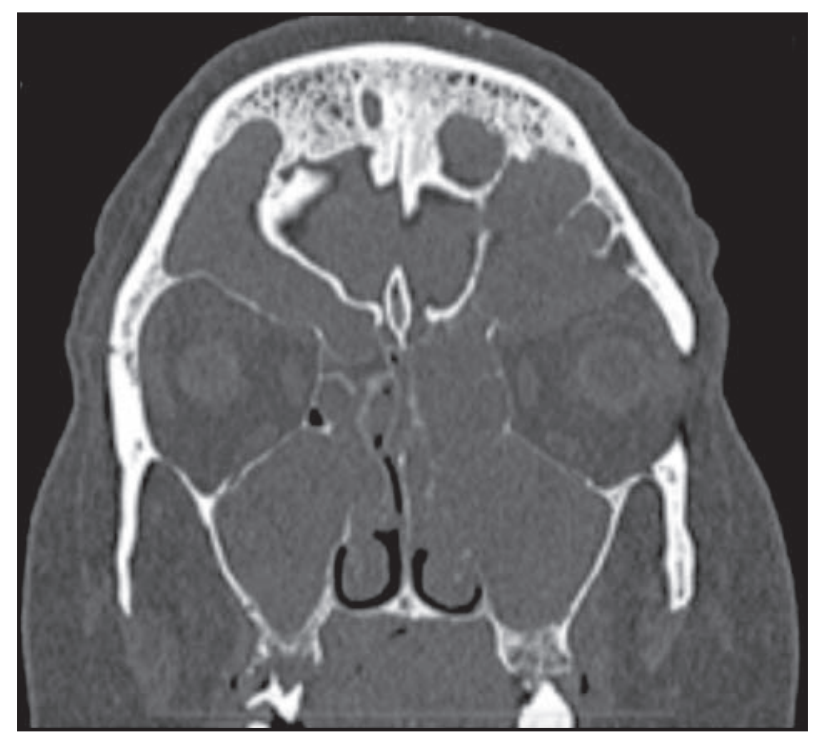

Figura 1. Tomografía computarizada corte coronal en ventana ósea. Se observan mucoceles frontoetmoidales bilaterales. Paciente con rinosinusitis crónica poliposa. 
de drenaje más estrechos, los que tendrían zonas de mayor contacto mucoso. El origen de los mucoceles se clasifican en primarios 0 secundarios, siendo los primarios aquellos en que ocurren de novo, sin antecedente de injuria, mientras que los secundarios tienen una causa traumática, tumoral 0 iatrogénica ${ }^{3}$. La continua producción de mucosidad dentro del seno obstruido genera la formación de una estructura quística revestida de mucosa respiratoria que se expande gradualmente, remodelando y erosionando el hueso vecino.

La proporción entre las distintas comorbilidades que originarían un mucocele varía según la literatura, donde predomina el porcentaje de causas secundarias. A nivel nacional se describe que el $50,1 \%$ de los mucoceles estuvo asociado a rinosinusitis crónica; $2,2 \%$ a displasia osteofibrosa y $2,2 \%$ a trauma maxilofacial ${ }^{7}$. A nivel internacional se ha reportado la presencia de una cirugía de cavidades paranasales previas entre $19,6 \%$ hasta $77,5 \%$ de los $\operatorname{casos}^{3,6,8}$. La presencia de mucoceles en población pediátrica es poco común y es altamente sospechosa de fibrosis quística, aunque también se puede presentar en casos de rinosinusitis crónica y trauma maxilofacial ${ }^{9}$.

Se ha demostrado que el epitelio del mucocele no pierde las características típicas de una mucosa respiratoria. Hay una expresión aumentada de prostaglandina $\mathrm{E}_{2}\left(\mathrm{PGE}_{2}\right)$ y macrófagos por sobre $40 \%$ respecto a lo normal, como también se ha asociado un incremento de expresión de interleuquinas (IL) 12, IL-2, colagenasa e interferón gamma ${ }^{10}$. Estos cambios bioquímicos proinflamatorios estimularían la reabsorción osteoclástica. También las fuerzas mecánicas, por el aumento de presión dentro de la cavidad del mucocele, inducirían una remodelación ósea a partir de estrés, lo que juntos podrían explicar los cambios óseos ${ }^{3,4}$.

El contenido de los mucoceles puede infectarse, ocasionando un piomucocele. Esta infección es polimicrobiana, principalmente por bacterias anaeróbicas provenientes de la flora orofaríngea como Peptostreptococcus sp, Prevotella sp, Porphyromona sp, Fusobacterium sp. También se encuentran bacterias aeróbicas como Staphylococcus aureus, Streptococcus alfa hemolítico y Haemophilus $\mathrm{sp}^{11}$.

\section{CLÍNICA}

Los mucoceles pueden permanecer creciendo de forma asintomática por mucho tiempo y pueden presentarse súbitamente con alguna complicación. Los principales síntomas dependen de dónde se desarrollan, ya que son producto de la expansión y remodelación ósea que ocasionan. La erosión de la órbita y de base de cráneo puede ocurrir hasta en $83,3 \%$ y $55,5 \%$ de los casos respectivamente ${ }^{3,6}$.

En cuanto a síntomas, los pacientes con presentación frontoetmoidal pueden desarrollar cefalea frontal, aumento de volumen o asimetría facial, también pueden tener manifestaciones oftalmológicas como reducción de agudeza visual, proptosis 0 alteración de la oculomotilidad. Se describe también en otras ubicaciones presión maxilofacial, obstrucción nasal, dolor periorbitario como también pacientes asintomáticos. Hallazgo de proptosis y diplopía se puede encontrar hasta en $83 \%$ y $45 \%$ de los casos respectivamente. La extensión intracraneal también puede llevar a meningitis y fístula de líquido cefalorraquíde $0^{8,12}$. En Chile, Nazar y cols describen sus hallazgos: Ios mucoceles frontoetmoidales se presentan en $51 \%$ de los casos con aumento de volumen ocular y en $41,4 \%$ con cefalea. Los maxilares se presentan en $57 \%$ con rinorrea y $28,6 \%$ con obstrucción nasal 0 descarga posterior. En cuanto a los esfenoidales, presentan cefalea y rinorrea ${ }^{13}$. Si se infecta el contenido desarrollando un piomucocele, la presentación clínica puede variar a cuadros similares a rinosinusitis aguda con potencial extensión a sitios vecinos: como órbita generando abscesos subperiósticos ${ }^{14}$ e intracraneano con meningitis y abscesos cerebrales ${ }^{15}$.

\section{DIAGNÓSTICO}

El diagnóstico de mucocele está basado en la anamnesis, examen físico y hallazgos radiológicos. La historia de alguna patología o intervención que pudiera orientarnos a la formación de un mucocele deben tenerse en cuenta, como rinosinusitis crónica, trauma maxilofacial, cirugía de cavidades paranasales previas. El tiempo entre la injuria sinusal previa y el diagnóstico del mucocele varía según la etiología, para la cirugía endoscópica funcional el promedio es 
de 5,3 años, para el trauma maxilofacial es de 17,7 años y para cirugía de cavidades paranasales es de hasta 18,1 años ${ }^{8}$. Dentro de la evaluación radiológica de los mucoceles, la tomografía computarizada (TC) y la resonancia magnética (RM) juegan roles importantes. La radiografía de cráneo no tiene utilidad para diferenciar de otras patologías y sólo podría llegar a mostrar velamiento y expansión del seno afectado.

La TC muestra de mejor manera los cambios óseos de las paredes de las CPN, las que pueden estar expandidas, adelgazadas 0 ausentes. El seno afectado se observa completamente velado por contenido de baja densidad en la mayoría de los casos. La presencia de aire dentro del quiste excluye el diagnóstico de mucocele. Con el uso de medio de contraste, realza el contorno del mucocele, sin realce central ${ }^{16,17}$. El realce del contorno muy marcado podría orientar a un piomucocele $e^{18}$ (Tabla 2). La densidad del contenido del mucocele puede ir de 10 a 18 unidades de Hounsfield (HU) si es una lesión reciente. Cuando la lesión es crónica, presentan de 20 a $40 \mathrm{HU}^{19}$.

La RM tiene mejor rendimiento para diferencias mucoceles de otras lesiones de tejidos blandos como también para evaluar las interfaces entre mucoceles y las estructuras intraorbitarias 0 intracraneanas. Una RM con uso de medio de contraste es útil para distinguir mucoceles, que realzan su periferia, versus neoplasias que típicamente tienen un realce de toda la masa sólida. Los mucoceles en la secuencia ponderada en T1 típicamente captan nula o escasa señal en su interior por el alto contenido de agua que contienen. En T1 con gadolinio podría captar señal la periferia del mucocele si está presente aún el mucoperiostio contiguo ${ }^{20}$. En la secuencia T2 tiene alta intensidad por el alto contenido de agua ${ }^{18}$. Las características de los mucoceles a la RM evolucionan con el tiempo en relación con la concentración proteica dentro del quiste. Eventualmente tendrán un aumento en la intensidad de T1 con disminución de la señal en T2 debido a un aumento del contenido proteico $0^{3,19}$. La intensidad de la secuencia T2 puede eventualmente desaparecer (Tabla 3). En caso de que las secreciones sean muy espesas, puede incluso no haber señal en T1 ni en T2, produciendo señal de vacío ${ }^{1}$. En caso de mucoceles pequeños, podría ser necesario para su evaluación únicamente una TC sin contraste. Pero si hay mucoceles grandes con compresión significativa de estructuras adyacentes, se recomienda TC y $\mathrm{RM}^{18}$.

\section{TRATAMIENTO}

El único tratamiento efectivo es quirúrgico. El objetivo de la cirugía es abrir la cavidad del

Tabla 2. Características a la tomografía computarizada

\begin{tabular}{|ll|}
\hline Tomografía computarizada & Características \\
\hline Sin uso de medio de contraste & $\begin{array}{l}\text { Paredes de cavidades paranasales expandidas, adelgazadas o ausentes. Seno comprometido com- } \\
\text { pletamente velado }\end{array}$ \\
\hline Con uso de medio de contraste & Realza el contorno del mucocele, sin realce central \\
\hline
\end{tabular}

Tabla 3. Características a la resonancia magnética

\begin{tabular}{|ll|}
\hline Secuencia & Características \\
\hline T1 & $\begin{array}{l}\text { Captan nula o escasa señal en su interior por el alto contenido de agua que contienen. En mucoceles más crónicos } \\
\text { podría haber captación de señal por aumento proteico }\end{array}$ \\
\hline T1 con gadolinio & Podría captar señal la periferia del mucocele si está presente aún el mucoperiostio \\
\hline T2 & Alta intensidad por alto contenido de agua. En mucoceles crónicos puede eventualmente desaparecer la señal \\
\hline
\end{tabular}


mucocele marsupializándolo con una adecuada ventilación y drenaje del seno comprometido. Una vez que se logre adecuada ventilación del seno, éste recuperará su mucosa normal². Los abordajes se pueden clasificar en abiertos, endoscópicos 0 combinados.

Clásicamente el abordaje abierto fue considerado el gold standard, pero se asocia a morbilidad significativa, deformidad facial y alta tasa de recurrencia ${ }^{1,13}$. Con los avances en instrumentos endoscópicos, cámaras y navegación guiada por imágenes, la tendencia de abordaje actual es a través de endoscopía nasal realizando una intervención funcional, mínimamente invasiva, sin cicatrices faciales, menos traumática, con menor tasas de morbilidad y evitando la recurrencia ${ }^{1,8,12,13,21}$. Además, el abordaje endoscópico facilita el examen endoscópico de la cavidad paranasal comprometida. El seno frontal es el que más limitaciones quirúrgicas podría llegar a presentar para realizar una cirugía completamente endoscópica, principalmente en mucoceles muy lateralizados, cuando hay abundante fibrosis o anatomía desfavorable, debiendo considerar caso a caso el abordaje ${ }^{21-23}$. La recurrencia de los mucoceles tratados con cirugía endoscópica funcional varía entre las di-

\section{BIBLIOGRAFÍA}

1. Georgalas C, Fokkens W. Acquired Noninfectious, Nonneoplastic Disorders of the Nose and Paranasal Sinuses. In: Georgalas C, Fokkens W, eds. Rhinology and Skull Base Surgery. Stuttgart: Georg Thieme Verlag; 2014. doi:10.1055/b-0034-78008.

2. Martel-Martín M, Gras-Cabrerizo JR, BotheGonzález C, Montserrat-Gili JR, De Juan-Delago M, Massegur-Solench H. Análisis clínico y resultados quirúrgicos en 58 mucoceles nasosinusales. Acta Otorrinolaringol Esp 2015; 66: 92-97. doi:10.1016/j.otorri.2014.06.002.

3. Devars Du Mayne M, Moya-Plana A, Malinvaud D, LacCourreye 0, Bonfils P. Sinus mucocele: Natural history and long-term recurrence rate. Eur Ann Otorhinolaryngol Head Neck Dis 2012; 129: 125-30. doi:10.1016/j.anorl.2011.10.002.

4. Obeso S, Luis Llorente J, Pablo Rodrigo J, Sánchez R, Mancebo G, Suárez C. Paranasal sinuses ferentes series, siendo de $0,9 \%$ a $8,9 \%$ a 4 años de seguimiento ${ }^{24}$. Los abordajes abiertos tienen históricamente una tasa de recurrencia de hasta $25 \%$ en 4 años ${ }^{3,8,21}$. Actualmente la indicación de abordaje abierto es limitado.

\section{CONCLUSIÓN}

Los mucoceles son lesiones benignas quísticas con potencial expansivo muy importante cuya ubicación más frecuente es frontoetmoidal. La mayoría de los mucoceles se originan a partir de injuria sinusal previa como rinosinusitis crónica, cirugía de CPN previa o trauma. Desde que se originan hasta que producen síntomas pueden pasar muchos años. Las principales manifestaciones son de acuerdo con su extensión, siendo las más frecuentes proptosis y diplopía. Es indispensable para el estudio contar con TC y si corresponde RM, según si es necesario evaluar extensión. El tratamiento es quirúrgico. El tratamiento endoscópico mínimamente invasivo ha desplazado al abordaje abierto por su menor comorbilidad, menor implicancias estéticas, menor recurrencia y mejor exposición para controles endoscópicos posteriores.

mucoceles. Our experience in 72 patients. Acta Otorrinolaringol Esp 2011; 60: 332-9. doi:10.1016/s2173-5735(09)70153-x.

5. González C, Fonseca $X$, Napolitano $C$. Mucocele de cornete medio ( Concha bulosa ): Reporte de un caso. Rev Otorrinolaringol Cir Cabeza Cuello 2005; $131-4$.

6. Barreto Plantier D, Neto DB, De Rezende Pinna F, Voegels RL. Mucocele: Clinical Characteristics and Outcomes in 46 Operated Patients. 2019. doi:10.1055/s-0038-1668126.

7. Osorio J, Muñoz D, Pardo J, Rahal M. Mucoceles de cavidades paranasales. Experiencia en el Hospital Barros Luco-Trudeau. Rev Otorrinolaringol Cir Cabeza Cuello 2014; 74: 109-14.

8. Scangas GA, Gudis DA, Kennedy DW. The natural history and clinical characteristics of paranasal sinus mucoceles: a clinical review. Int Forum Allergy Rhinol 2013; 3: 712-7. doi:10.1002/alr.21178.

9. Nicollas R, Facon F, Sudre-Levillain I, Forman C, Roman S, Triglia JM. Pediatric paranasal sinus 
mucoceles: Etiologic factors, management and outcome. Int J Pediatr Otorhinolaryngol 2006; 70: 905-8. doi:10.1016/j.ijporl.2005.10.002.

10. Lund VJ, Henderson B, Song Y. Involvement of cytokines and vascular adhesion receptors in the pathology of fronto-ethmoidal mucocoeles. Acta Otolaryngol 1993; 113: 5406. doi:10.3109/00016489309135860.

11. Brook I, Frazler EH. The microbiology of mucopyocele. Laryngoscope 2001; 111: 1771-3. doi:10.1097/00005537-200110000-00020.

12. Aggarwal S, Bhavana K, Keshri A, Kumar R, SRIVAStava A. Frontal sinus mucocele with orbital complications: Management by varied surgical approaches. Asian J Neurosurg 2012; 7: 135. doi:10.4103/1793-5482.103718.

13. Nazar R, Naser A, Pardo J, Fulla J, RodríguezJorge J, Delano PH. Manejo endoscópico de mucoceles de senos paranasales: Experiencia en 46 pacientes. Acta Otorrinolaringol Esp 2011; 62: 363-6. doi:10.1016/j.otorri.2011.04.004.

14. Chang-Wel Ding; QIU-ShI Wang, MS; QI-Yong Guo, MD; Jun Zhang, MD; Zhen-Hal Wang M. CT and MRI features in adult patients with orbital subperiosteal abscess secondary to paranasal sinus mucocele. Ear Nose Throat J 2017; 96: 258-62. doi:10.1177/014556131709600701.

15. Malard 0, Gayet-Delacroix M, Jegoux F, Faure A, Bordure P, De Montreuil CB. Spontaneous sphenoid sinus mucocele revealed by meningitis and brain abscess in a 12-year-old child. Am J Neuroradiol 2004; 25: 873-5.

16. Van Tassel P, Lee Yy, Jing BS, De Pena CA. Mucoceles of the paranasal sinuses: MR imaging with CT correlation. Am J Neuroradiol 1989; 10 : 607-612.

17. Huang B, Lloyd K, Del Gaudio JM, Jablonowksi E, Hudgins P. Failed Endoscopic Sinus Surgery: Spec- trum of CT Findings in the Frontal Recess. Radiographics 2009; 7510. doi:10.1148/rg.291085118.

18. Koch BL, Hamilton BE, Hudgins PA, Harnsberger HR. Diagnostic Imaging. Head and Neck $3^{\text {rd }}$ ed. Elsevier Ltd; 2016.

19. Lloyd G, Lund VJ, Savy L, Howard D. Optimum imaging for mucoceles. J Laryngol Otol 2000; 114: 233-6. doi:10.1258/0022215001905238.

20. Edelman RR. Clinical Magnetic Resonance Imaging. Saunders Elsevier; 2006. https://books. google.cl/books?id=Y8UhAQAAMAAJ\&q=07216 03068\&dq=0721603068\&hl=es-419\&sa=X\&ved =0ahUKEwinz4K2xrXjAhXfKrkGHb81DW8Q6AE IKTAA. Accessed July 14, 2019.

21. Courson AM, Stankiewicz JA, Lal D. Contemporary management of frontal sinus mucoceles: A metaanalysis. Laryngoscope 2014; 124: 378-86. doi:10.1002/lary.24309.

22. Chiu AG, Palmer JN, Adappa ND. Atlas of Endoscopic Sinus and Skull Base Surgery. In: Atlas of Endoscopic Sinus and Skull Base Surgery 2018; 171-176. doi:10.1016/C2015-0-01193-5.

23. Dos Santos PL, Chihara LL, Alcalde LFa, Masalskas BF, Sant'ana E, Faria PEP. Outcomes in Surgical Treatment of Mucocele in Frontal Sinus. $J$ Craniofac Surg 2017; 28: 1702-8. doi:10.1097/ SCS.0000000000003224.

24. HAR-EL G. Endoscopic management of 108 sinus mucoceles. Laryngoscope 2001; 111(12): 21314. doi:10.1097/00005537-200112000-00009.

Correspondencia: Constanza Valdés P. 